\title{
Defining health catchment areas in Jeddah city, Saudi Arabia: an example demonstrating the utility of geographi- cal information systems
}

\author{
Abdulkader A. Murad \\ Department of Urban and Regional Planning, Faculty of Environmental Design, King Abdulaziz \\ University, P.O. Box 80210, Jeddah 21589, Saudi Arabia
}

\begin{abstract}
A tool, based on a geographical information system (GIS) approach, has been developed for the location and organization of public health care centers in Jeddah city, Saudi Arabia. The potential of GIS for visualizing and modeling available information on health-care has been utilized to produce a "best-fit application". This paper identifies and deals with the manner in which GIS can successfully be used for evaluating the demand and supply of health care facilities as well as be applied for defining health catchment areas. The study uses the essential achievements gained to analyze the advantages of GIS for health care planning and the location of health care centers. Some specific GIS tools, as well as single and multiple spatial search functions, are presented.
\end{abstract}

Keywords: geographical information systems, health centers, catchment areas, straight-line allocation function, Saudi Arabia.

\section{Introduction}

Local health authorities are continuously faced with many and various challenging tasks and functions. The monitoring of changes in health services demand and supply is an important area where large sets of data need to be managed, e.g. the size of populations to be served, the number of physicians and dentists available, and access to other medical specialists at each service area. Computers are needed to store and access this amount of data in an effective way, while a geographical information system (GIS) approach lends itself to the accurate handling of such information. A growing number of studies are seen to start exploring the advantages of using

Corresponding author:

Abdulkader A. Murad

Department of Urban and Regional Planning

Faculty of Environmental Design, King Abdulaziz University

P.O. Box 80210, Jeddah 21589, Saudi Arabia

Tel./Fax +96626952247

E-mail: amurad25@hotmail.com
GIS in the planning of health care and many researchers create and link statistical models with a GIS approach to better understand the spatial reasons for health-related incidents and/or diseases.

GIS is a well-suited approach to evaluating patient access to health care facilities. The defining of catchment areas for health care can be done in different ways for example, health authorities evaluate the distribution of supply versus demand within a region, often expressed as a ratio of population to health resources (Luo, 2004). Or health authorities use a predefined area such as a census country division or a minor civil division for the purpose of identifying the catchment area. The advantage of the latter approach is that it is simple and straightforward as information regarding physicians and populations are readily available. It should also be noted that the catchment areas are not just theoretical but can in fact be located in the real world. On the other hand, this approach has been criticized by many researchers who point out that it rests on the assumption that: (i) people within a region have 
equal access to physicians within that same region, and (ii) people do not venture beyond their own region to seek care (Luo, 2004). It has been argued that the first assumption may not always be true and that spatial disaggregated data are required for every health care facility location to know the socalled provider-to-population ratio. This information is useful for gross comparison of supply between geopolitical units or service areas and is used by policy analysts to set minimal standards of local supply and to identify underserved areas. In addition, the classic problem of spatial border crossing, which can be substantial for small geographies such as urban census tracts (Guagliardo et al., 2004), is often ignored. To overcome these drawbacks, this paper proposes and discusses a more analytical method for defining health catchment areas based on the distance to the nearest health center. This is based on the straight-line allocation function (SLA) of GIS software, briefly introduced here.

The assessment and definition of catchment areas represent important GIS usages in health care planning. When considering the location and use of health care centers and other facilities, GIS overlay functions are applied. In this way, information on the general socioeconomic structure, census boundaries and service area definitions can be presented along with patient data such as age, sex and home address.

GIS analytical functions can be applied to predict unknown values of any geographical point data such as elevation or disease concentration. Kriging is a powerful GIS function which has the capability of producing prediction surfaces and also providing a measure of the degree of accuracy of the prediction made (Johnston et al., 2001). The GIS software ArcGIS (ESRI, Redlands, Ca, USA), for example, has modules, or extensions, that deal with data prediction issues, e.g. the so-called ArcGIS-geostatistical analysis module which has several useful prediction functions, the inverse distance-weighted function being one of them. The latter can be used for predicting values of data sets from a limited number of sample data points assuming that the variable decreases in dependence with the distance from its sampled location (McCoy and Johnston, 2001). There are also other GIS techniques, such as overlay analysis, buffering, network and allocations functions, which are important in public health research and which can be implemented for investigating resource distribution between services and the surrounding population (Murad, 2004). Some of these GIS functions are applied and discussed in this paper which addresses the possibilities of the GIS approach for the effective management of health care centers in Jeddah city, Saudi Arabia.

\section{Material and methods}

\section{Study area}

This paper concerns the different types of health care facilities available to the residents of Jeddah city in Saudi Arabia. In addition to governmental general-care health centers and specialized hospitals, there are also private facilities which also include health care centers and hospitals. Although physicians can thus either be working in the governmental or in the private health care services, we only address and analyze the situation from the point of view of the governmental health care centers. The reason is that the public centers, in contrast to the private facilities, are forced to match available resources to the known demand.

\section{General approach and technical support}

The first issue was to construct a GIS model that describes the existing situation in Jeddah city with regard to the supply of human resources for health services. This model is important for health planners not only because it provides a visual view of the facilities and services at their disposal, but since it also shows the problem areas and identifies the parts of the city which requires more detailed health research. Several types of data were used to construct this model of the existing health services in 
Jeddah city. All health centers were plotted on a GIS as a point features map and classified based on the type of service supplied, i.e. (i) physicians (general practitioners (GPs)), (ii) family physicians, (iii) dentists, (iv) nurses, (v) midwives, (vi) pharmacists/pharmacologists, (vii) laboratory technicians, (viii) X-ray technicians, (ix) administrators, $(\mathrm{x})$ general staff, and (xi) non-classified support staff.

The GIS software packages such as ArcGISArcInfo and ArcGIS-Arcview (ESRI, Redlands, Ca, USA) include many functions and tools designed for health care studies. These functions can be used for the visualization of health data, spatial analysis and modeling. They are crucially important in any health-oriented study as they enable better decisionmaking by providing health status and needs for an area or region from a spatial point of view. GISbased analysis and modeling functions can be classified according to data model types (vector or raster data). The density function, for instance, can be used as point data for the location of health care centers, for the estimation of spatial variations in disease risk and for a spatial demonstration of point-pattern intensity (Gatrell and Senior, 1999). The output of the density function is more useful for pattern recognition than for the location of individual features and, particularly, for mapping areas of different sizes such as census tracts or counties. Two main methods are used for calculating density in the ArcGIS software. The first one simply counts the features within a search radius, while the second one is a weighted method used as a mathematical function (e.g. the Kernel function) to emphasize features closer to the centre of raster cells (McCoy and Johnston, 2001). Health care data such as location of patients can be analyzed by this function to identify the spatial variation of these patients in any area (Murad, 2007).

In addition to apply GIS for defining spatial classification of health supply, it can also be used to for multiple data classification or so-called multiple queries. This type of GIS function reveals features satisfying a criterion with more than one parameter, e.g. to identify health centers with many physicians but no dentist, or with inadequate number of general staff. Multiple queries can be made in GIS using logical operations dealing directly with the database allowing the user to identify and select features by a special set of criteria (Chou, 1997). In most GIS applications, features are identified and selected from the database and highlighted on the map. Such selected features can be saved in a new coverage for further analysis. Presently, multiple queries were used identify health care centers with more than three physicians which are owned by the health authorities. This type of search was used to select health care centers providing a broadly based service, but not housed in a public building, in order to facilitate decisions regarding which health care centers should be given priority to move into new public buildings when such premises have been erected.

A database and model was created with the aim of supporting the health authority of Jeddah city in deciding the best size of their catchment areas. To produce it, data were gathered about the health care centers, i.e. location of the centers, existing service boundaries, statistics about existing demand (the registered patients) and supply (the number of physicians working in each center). Additionally, other important information, i.e. the road network in Jeddah city and city districts' boundaries with their relevant population data, was added. The ArcGIS software, used for the construction of the database and the capture and manipulation of the data, is based on three packages with different functions and tools: (i) the ArcCatalog for managing and holding the spatial data and data base designs as well as for recording and viewing meta-data (Ormsby et al., 2004); (ii) the ArcMap for any mapping and editing tasks as well as for map-based analysis; and (iii) the ArcToolbox for data conversion and geo-processing tasks.

\section{Modeling bealth catchment areas}

Modeling generally relies on advanced mathematics and various GIS functions and tools are needed in this approach. Therefore, prior to undertaking 
the construction of a workable model, one needs to identify the method that would best lend itself to establishing a useful GIS-based catchment area. As revealed by several recent studies, the definition of health catchment areas is a major task in any local health care planning. Shortt et al. (2005) estimated health regions in northern Ireland using a multidimensional approach combined with GIS, while Bullen et al. (1996) applied GIS for defining catchment areas of the health centers in West Sussex, UK. Eight methods, or approaches, have been described (Shortt et al., 2005) based on:

(i) a $75 \%$ percentage catchment;

(ii) a $85 \%$ percentage catchment;

(iii) a market-share catchment;

(iv) the nearest feature catchment using straightline distances;

(v) the nearest health center's catchment according to road travel distances;

(vi) a patient-to-surgery mean distance catchment;

(vii) an administrative catchment (using the 10-mile radius definition); and

(viii) the perceived catchment (as decided by the local health authorities themselves).

Each of these catchments approaches can be produced with different GIS functions and modules. The present application is based the $4^{\text {th }}$ approach above to define the health catchment area. To obtain this type of catchment area, the GIS functions on the vector or raster data sets were used applying the SLA function on the health centers shape file. The idea of SLA is to measure the straight-line distance from each raster cell to the closest source and to assign to each cell the value of the source to which it is closest (McCoy and Johnston, 2001). The SLA function is located within ArcGIS spatial analysis software extension, a raster-based analytical software providing a wide range of useful tools for performing spatial analysis such as identifying spatial relationships between data sets, locating suitable sites, or finding the best route through a landscape. There are several potential applications of the SLA function such as, for example:

(i) identifying customers/clients served by a serv- ice or stores;

(ii) identifying the closest hospital or health center;

(iii) finding areas with a shortage of fire hydrants; and

(iv) locating areas not served by a chain of supermarkets.

Provided that there are data available about population size for every single district, we used the GIS overlay analysis functions designated as "intersect" for the new catchment areas and for the city districts' coverage. The intersect function is equivalent to the Boolean "AND" operation and if it is used on two coverages, only the portion of the input coverage (city districts) that falls inside the intersect coverage (new catchment area) remains in the output coverage (Chou, 1997).

\section{Results}

A model how the health centers are classified based on the number of physicians is shown in Figure 1, while Figure 2 deals with the number of dentists. These figures provide a visual evaluation and comparison between health care centers based on their actual supply of human recourses. The features are presented in proportion to their relative impact and importance, i.e. features related to health data based on large population values are presented with large icons or symbols, while low population features are shown with smaller symbols.

Figure 3 gives the name and location of every health care centre in Jeddah city. It also shows the city district borders and it can be used as a guide to define the distribution of the population in the area. The health care centers have different numbers of physicians or dentists. Some centers such as the Alsafa Centre and the Gulail Centre engage many physicians, while in other ones, such as the Alsharafia Centre and the Althayuar Centre, their numbers are smaller. The situation is similar concerning the dentists. While some centers have two dentists, e.g. the Alazizia Centre, in others, e.g. the Alselamainah Centre and the Almarwah Centre, there are none at all. 


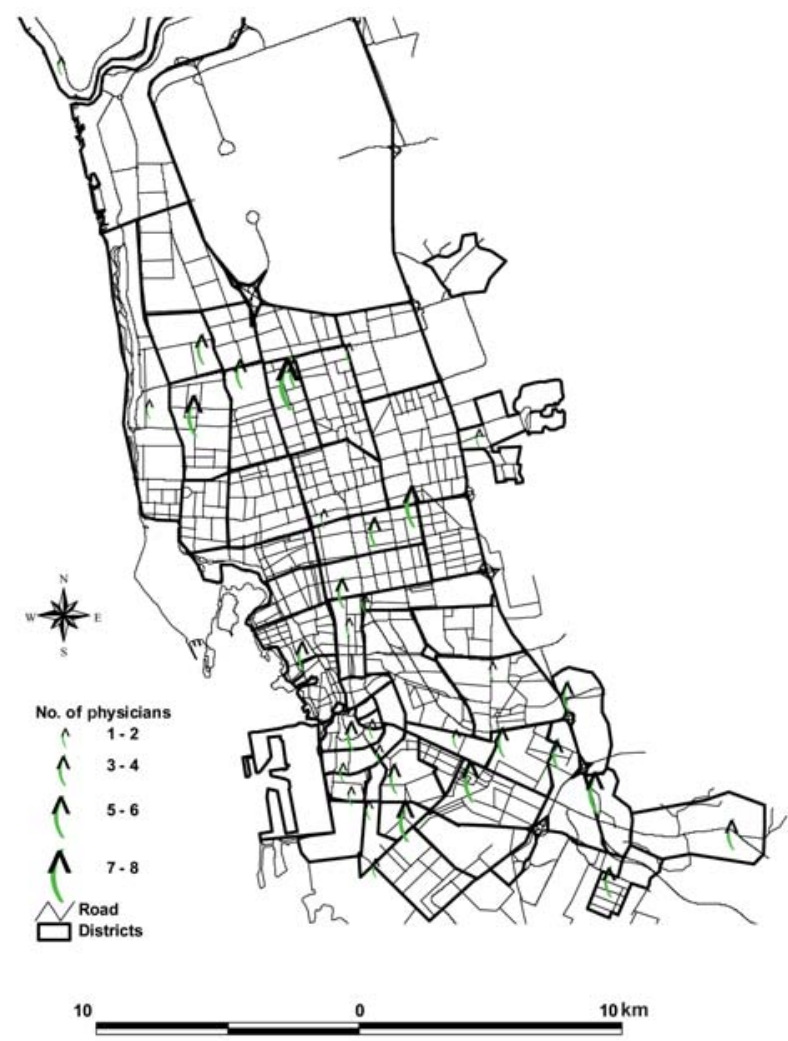

Fig. 1. Classification of health care centers based on the number of physicians.

Figure 4 describes the results of the multiple-query search and not only it can be seen that several health care centers in Jeddah city engage a large number of physicians, but also that they are located in rented buildings. Health care centers of this type are mainly located in the southern part of the city (e.g. the Ghulail and the Aljameaa centers). However, there are other centers located in the northern part of the city (e.g. the Alzahraa and the Alsalamah centers) with the same characteristics.

The multiple-search model was created to define the health care centers delivering a large and broadly based health care by family physicians and dentists (Fig. 5). Obviously, some of these centers, e.g. the Alrabwa, the Alzahraa and the Alnaeem centers, meet these requirements. However, other centers, e.g. the Alsafa and the Alsohaifa centers, do not engage any dentists or family physicians. The local

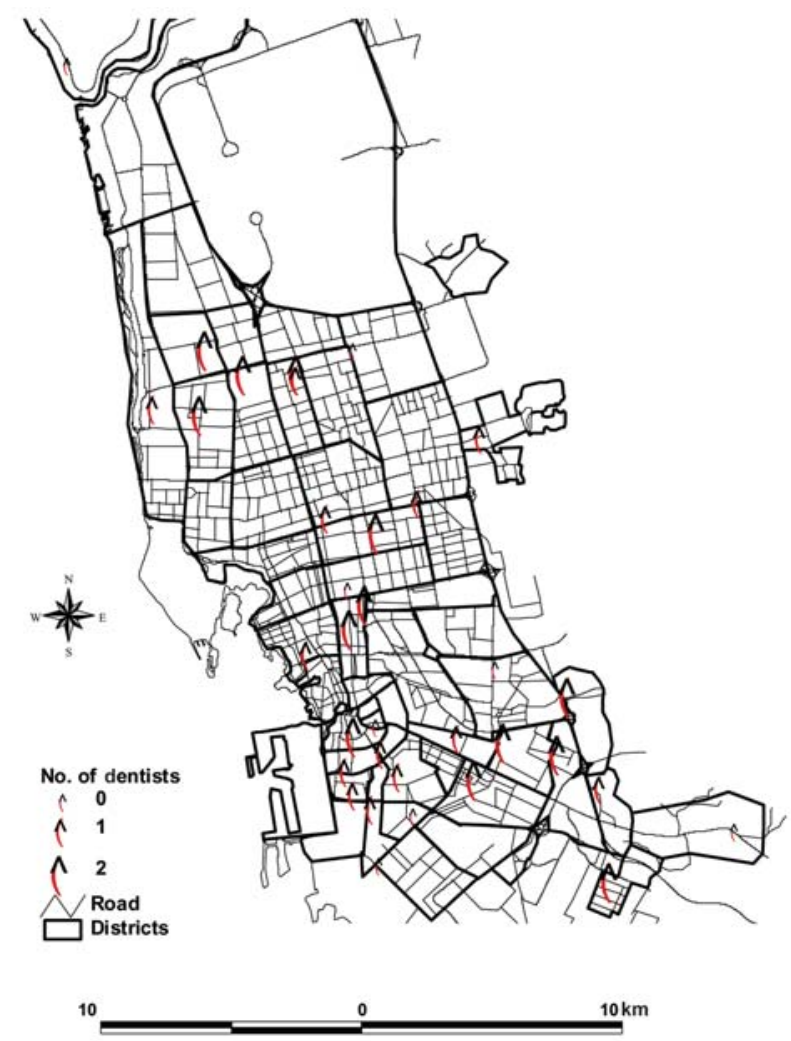

Fig. 2. Classification of health care centers based on the number of dentists.

health authority can therefore make use of this approach to find quick and clear answers about any issue related to the quality and the quantity of health care services delivered in Jeddah city.

Figure 6 displays a set of new catchment areas for the health care centers in Jeddah city produced by the SLA function and based on the closest-proximity approach. Each health care centre's location is assigned with this approach in mind and each person in its area is closer to the area's health care centre than to any other health care centre. In order to evaluate this output, existing health catchment areas, created by the local health authority (Fig. 7), were digitized as vector polygons and then transformed into a raster for analysis using a spatial analyst extension of the software employed. Finally, they were compared to the resulting catchment areas aiming at identifying the percentage of 


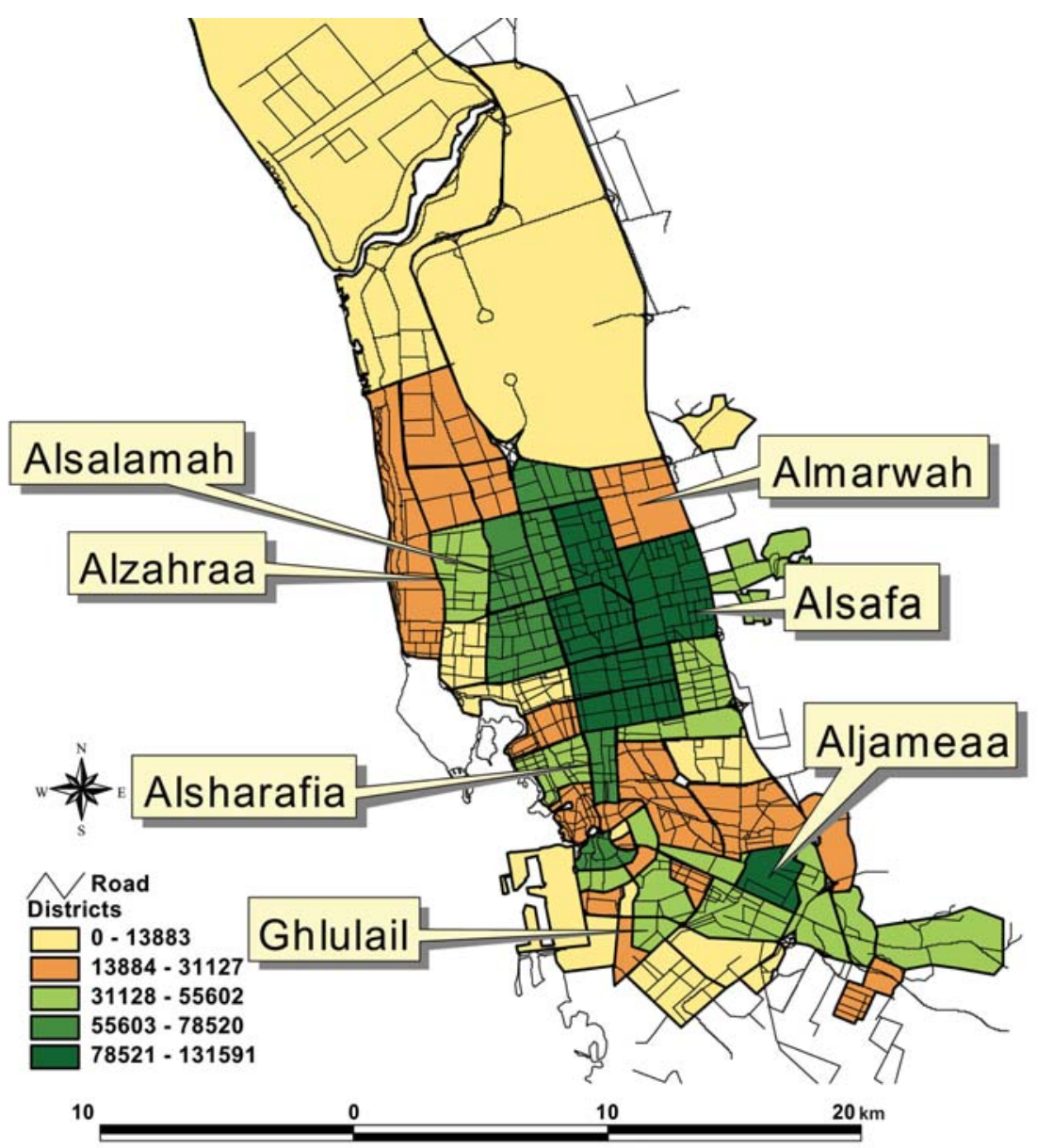

Fig. 3. The population distribution in Jeddah city districts.

changes that take place in every health catchment area. The total surface of every new service area is divided by the local authority catchment area and the results multiplied by 100 to give an index of change for the health catchment areas in Jeddah city. Figure 8 indicates that almost every existing health catchment area has been reduced and that the people inside them will as a consequence receive better health care services. The reduction in size will lead to a decrease of demand and health services required in every health care centre. The Alshate Centre's 1620 ha health care service area changed to a 640 ha one $(40 \%$ reduction) resulting from SLA functionbased analysis. The same situation is valid for the Alsafa and Alaziziyah centers.
Figure 9 displays the spatial distribution of the population inside every health catchment area. It is obvious that some, e.g. the Alsafa and the Banimalik centers, have a larger population sizes. According to the local standards, every physician should serve at least 2000 persons. This standard can be applied to calculate the number of physicians required in every health centre.

\section{Discussion}

The first step in health care research consists usually of establishing a health information chart visualizing where health centers are located and how they are staffed. This is useful in that it provides an 


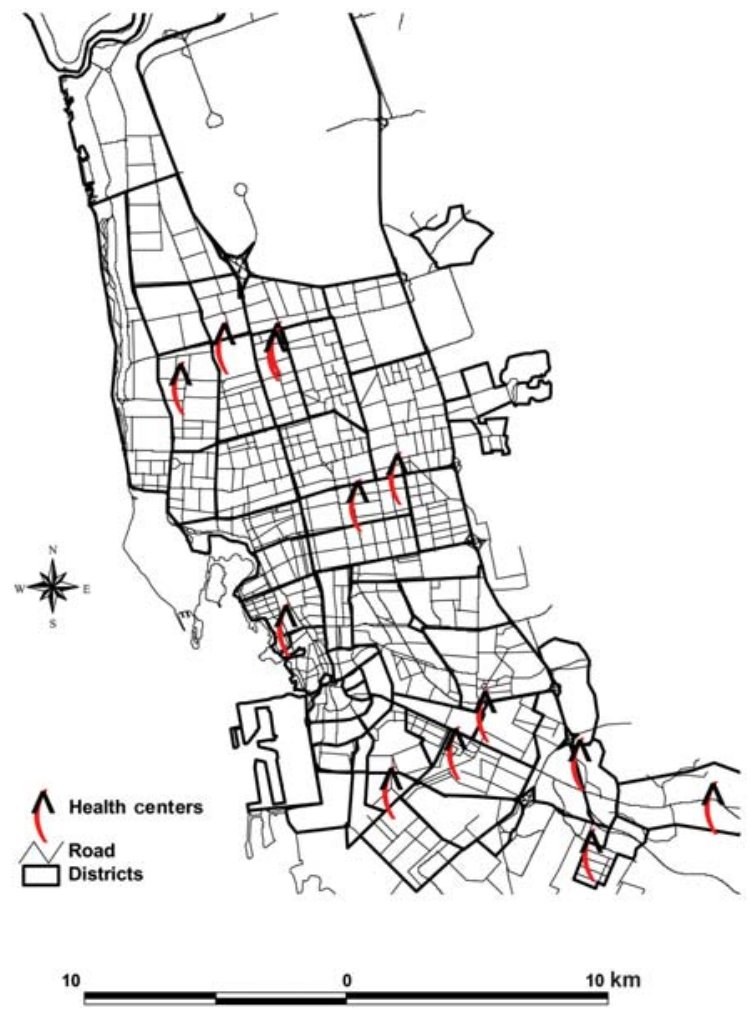

Fig. 4. Rented health care centers with more than 3 physicians.

overview of data distributions and possible spatial patterns. The type of health care that can be delivered at a health care centre depends on its organization, in particular on its human resources. Maps play an important role here and have traditionally been used to analyze associations between location, environment, and disease. Kumar (2004) has utilized them to study the factors governing geographic accessibility and effective location of basic public health care units. He has also applied location-allocation models to examine changing geographic access and the efficiency of basic public health care needs vis-a-vis private healthcare services in India (Kumar, 2004). Miller (2007), using a drinkingwater programme in a small local health department in Whatcom county, Washington, USA, recommends GIS users and other researchers to collaborate over the Internet.

The spatial distribution of population and the

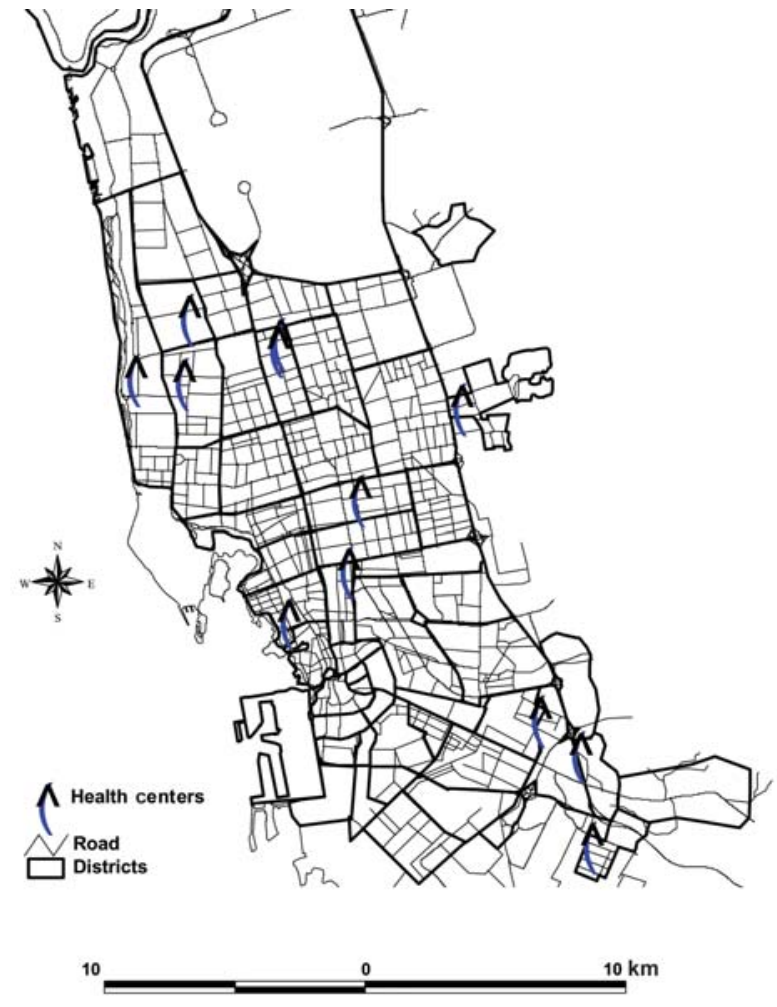

Fig. 5. Health care centers with family physicians and dentists.

location of health care services are related to key variables such as bed capacity and catchment distance in order to generate information vital for decision-making regarding the planning of health services in Uganda (Lwasa, 2007). Lee et al. (2007) suggest the development of a spatial information decision-making system in the State of Mississippi, USA, on the basis of GIS interfaces in order to direct injured people to the closest, most appropriate facility based on the nature of the problem, the resources at this facility, travel time, insurance coverage, staff and bed availability, etc. Leonardo et al. (2007) outline the benefits of using remote sensing from earthobserving satellites and GIS for health research in the Philippines as tools to improve the health services delivery system in this country. Kulldorff (1998) use statistical tests and GIS to define the purely spatial variation of disease risk, while Wilkinson et al. (1998) provide a list of different issues related to 


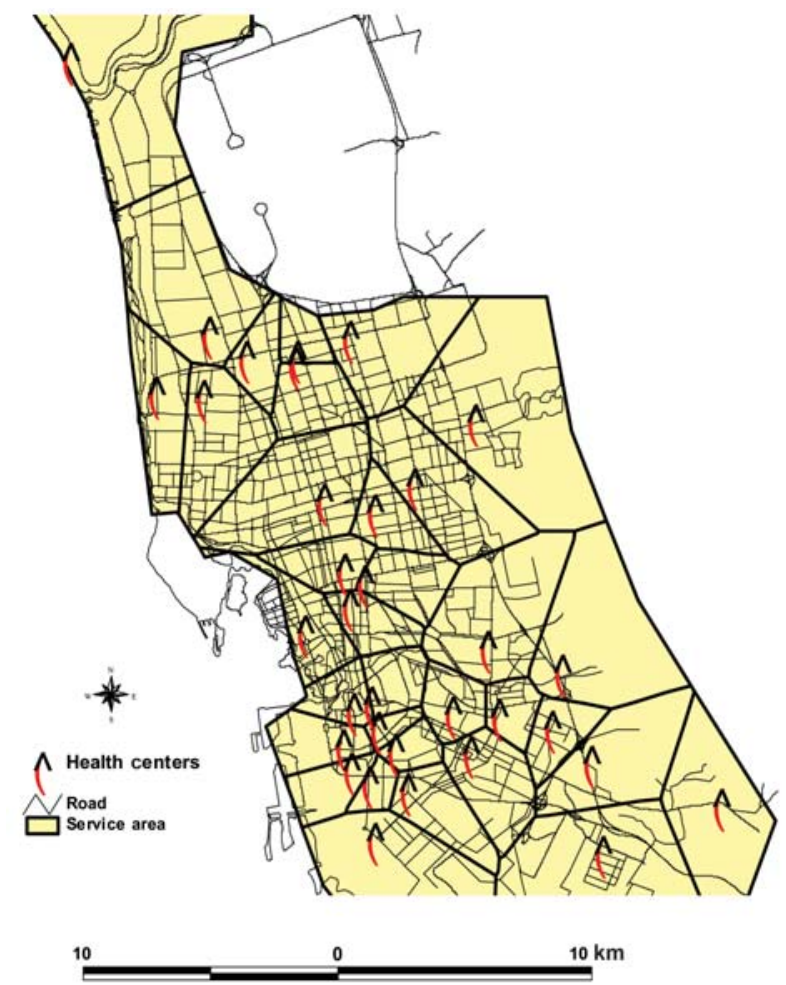

Fig. 6. Application of the SLA function for new catchment areas of health care centers.

health care analysis, e.g. the spatial distribution of health care facilities and the referral patterns to aid decisions about optimal location of health services. Kivell et al. (1990) discuss an example of using GIS for health service administration and Murad (2004) has applied GIS and a network analysis module to create a catchment area of a health centre in Makkah city, Saudi Arabia, based on demand and supply of health care. GIS is particularly well-suited to this challenge because of its spatial analysis and display capabilities (Jacquez, 2004; Rosero-Bixby, 2004; Waring et al., 2005; Mobley et al., 2006).

The variable size of the spatial units is an important issue in health data. Clearly, this information stands out when presented visually. However, it should be noted that the potential problem of dealing with limited numbers of people scattered over large areas can be more important to address than organizing smaller areas with larger populations. In

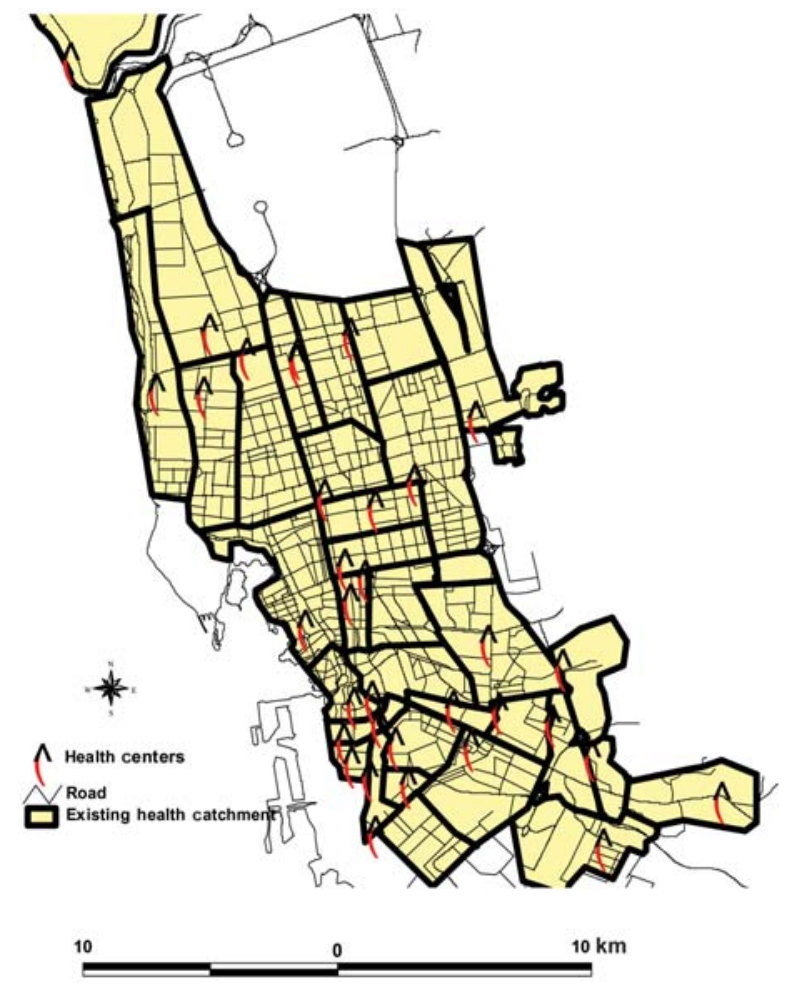

Fig. 7. Existing health care centre catchment areas.

addition, the visualization would not be so useful if the relative importance of what is presented would not be obvious to the viewer. It is therefore helpful that GIS permits the construction of maps that show features in proportion to their relative impact and importance. For example, features related to health data based on large population values are presented in GIS with large icons or symbols, while low population features are shown with smaller symbols. This is also useful for presenting the staffing of each centre. For example, it is immediately clear from Figures 1 and 2 that every centre has a different number of physicians and dentists. It is also evident, as seen in Figure 5, that the multiple-search model delivers quick and clear answers about any issue related to the quality and the quantity of health care services delivered in Jeddah city. Importantly, the reduction in size of almost every existing health catchment area (Fig. 8) will lead to a decrease of 


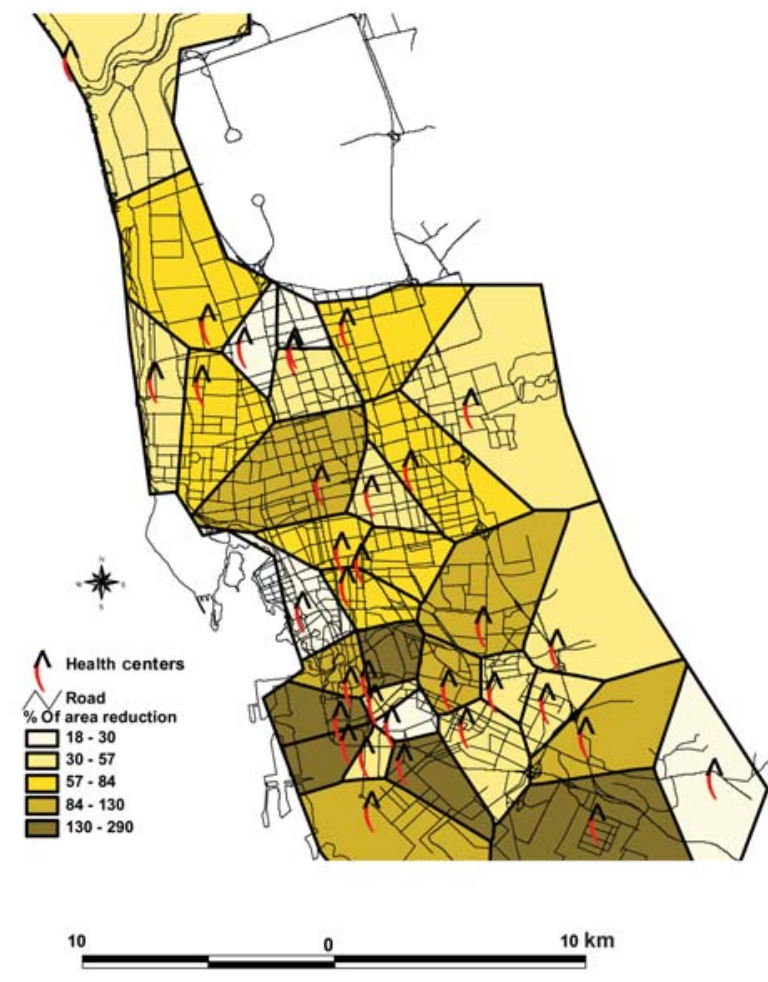

Fig. 8. Catchment area reductions based on the SLA function.

demand and health services required in every health care centre. Knowledge of the spatial distribution of the population in the health catchment area is useful to the health authorities as they can now adjust the need for health care based on reliable information. For example, this is the justification needed to provide catchment areas with larger populations the needed medical services. In fact, this type of output can be used by the local health authority to define the required health supply exactly in every health service area. Based on the calculated need of health care staff per person the health authority can adjust and re-allocate their supply of medical services in the different catchment areas.

In conclusion, the present paper demonstrates that a series of health care planning issues can be effectively handled and managed by existing GIS software packages. Specific GIS-based models, related to two main health-service planning aspects, have

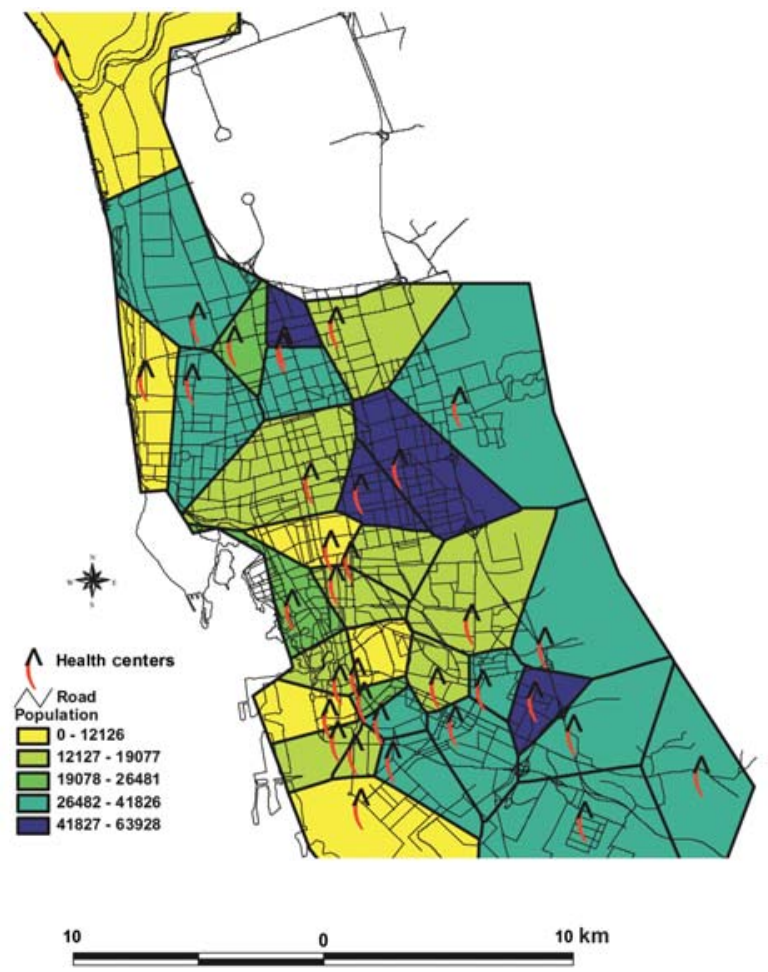

Fig. 9. Health demand in Jeddah City, Saudi Arabia.

been created for Jeddah city.

A remarkable difference in the amount of health supply of every center was found. Single and multiple spatial search GIS functions have been applied to produce a health supply model. Based on the SLA function, a new health service area model for every centre has been produced and then compared with the actual local health authority service area. It was noted that a considerable improvement of the size of the service areas could be achieved when implementing the SLA service area model for the city health centers.

\section{References}

Bullen N, Moon G, Jones K, 1996. Defining localities for health planning: a GIS approach. Soc Sci Med 42, 801-816. Chou Y-H, 1997. Exploring spatial analysis in geographic information systems. Onward Press, Santa Fe, NM, USA. 
Gatrell A, Senior M, 1999. Health and health care applications. In: Geographical Information Systems. P Longley, M Goodchild, D Maguire, D Rhind (eds). John Wiley \& Sons, New York, pp. 925-938.

Guagliardo M, Ronico C, Cheung I, Chacko E, Josef J, 2004. Physician's accessibility: an urban case of pediatric providers. Health Place 10, 273-283.

Jacquez GM, 2004. Current practices in the spatial analysis of cancer: flies in the ointment. Int J Health Geogr 3, 22.

Johnston K, Hoef J, Krivoruchko K, Lucas N, 2001. Using ArcGIS geostatistical analyst. Environmental Systems Research Institute, Redlands, CA, USA.

Kivell P, Turton B, Dawson B, 1990. Neighborhoods for health service administration. Soc Sci Med 20, 701-720.

Kulldorff M, 1998. Statistical methods for spatial epidemiology: tests for randomness. In: GIS and Health. A Gatrell, M Loytonen (eds). Taylor \& Francis, London, UK, pp. 49-62.

Kumar N, 2004. Changing geographic access to and locational efficiency of health services in two Indian districts between 1981 and 1996. Soc Sci Med 58, 2045-2067.

Lee JE, Sung JH, Ward WB, Fos PJ, Lee WJ, Kim JC, 2007. Utilization of the emergency room: impact of geographic distance. Geospatial Health 2, 243-253.

Leonardo LR, Crisostomo BA, Solon JAA, Rivera PT, Marcelo AB, Villasper JM, 2007. Geographical information systems in health research and services delivery in the Philippines. Geospatial Health 2, 147-155.

Luo W, 2004. Using a GIS-based floating catchment method to assess areas with shortage of physicians. Health Place 10, $1-11$.

Lwasa S, 2007. Geospatial analysis and decision support for health services planning in Uganda. Geospatial Health 2, 29-40.

McCoy J, Johnston K, 2001. Using ArcGIS spatial analyst:
GIS by ESRI. Environmental Systems Research Institute, Redlands, CA, USA.

Miller C, 2007. The introduction and expansion of GIS into a small local health department drinking-water program. J Environ Health 69, 35-40.

Mobley LR, Root E, Anselin L, Lozano-Gracia N, Koschinsky J, 2006. Spatial analysis of elderly access to primary care services. Int J Health Geogr 5, 19.

Murad A, 2004. Creating a GIS application for local health care planning in Saudi Arabia. Int J Environ Health Res 14, 185-199.

Murad A, 2007. A GIS application for modeling accessibility to health care centers in Jeddah city. In: GIS for Health and the Environment: Development in the Asia Pacific Region. C Lai, A Mak (eds). Springer, Berlin, Germany, pp. 57-70.

Ormsby T, Napoleon E, Burke R, Fesster L, Groessle C, 2004. Getting to know ArcGIS desktop. Environmental Systems Research Institute, Redlands, CA, USA.

Rosero-Bixby L, 2004. Spatial access to health care in Costa Rica and its equity: a GIS-based study. Soc Sci Med 58, 1271-1284.

Shortt N, Moore A, Coobes M, Wymer C, 2005. Defining regions for locality health care planning: a multidimensional approach. Soc Sci Med 60, 2715-2727.

Waring S, Zakos-Feliberti A, Wood R, Stone M, Padgett P, Arafat R, 2005. The utility of geographic information systems (GIS) in rapid epidemiological assessments following weather-related disasters: methodological issues based on the tropical storm Allison experience. Int J Hyg Environ Health 208, 109-116.

Wilkinson P, Grundy C, Landon M and Stevenson S, 1998. GIS in Public Health. In: GIS and Health. A Gatrell, M Loytonen (eds). Taylor \& Francis, London, UK, pp. 179189. 